\title{
Millimeter wave imaging: a historical review
}

\section{Roger Appleby, Duncan A. Robertson, David Wikner}

Roger Appleby, Duncan A. Robertson, David Wikner, "Millimeter wave imaging: a historical review," Proc. SPIE 10189, Passive and Active Millimeter-Wave Imaging XX, 1018902 (11 May 2017); doi:

$10.1117 / 12.2262476$

SPIE. Event: SPIE Defense + Security, 2017, Anaheim, California, United States 


\title{
Millimeter wave imaging: a historical review
}

\author{
Roger Appleby*a, Duncan A. Robertson ${ }^{\mathrm{b}}$, David Wikner ${ }^{\mathrm{c}}$ \\ ${ }^{a}$ InnovaSec Ltd., 212b West Malvern Road, Malvern, Worcs, WR14 4BA, UK; \\ ${ }^{\mathrm{b}}$ University of St Andrews, SUPA School of Physics \& Astronomy, St Andrews, Scotland; \\ ${ }^{\mathrm{c}}$ U.S. Army Research Laboratory, Adelphi, MD 20783, USA.
}

\begin{abstract}
The SPIE Passive and Active Millimeter Wave Imaging conference has provided an annual focus and forum for practitioners in the field of millimeter wave imaging for the past two decades. To celebrate the conference's twentieth anniversary we present a historical review of the evolution of millimeter wave imaging over the past twenty years. Advances in device technology play a fundamental role in imaging capability whilst system architectures have also evolved. Imaging phenomenology continues to be a crucial topic underpinning the deployment of millimeter wave imaging in diverse applications such as security, remote sensing, non-destructive testing and synthetic vision.
\end{abstract}

Keywords: Millimeter wave imaging, Review

\section{INTRODUCTION}

Passive Millimeter wave imaging was first discovered in the 1950s when work on components for radar provided receivers which were sensitive enough to detect the radiation naturally emitted and reflected from the environment. However these systems were very large and lacked sensitivity and it was only when good quality semiconductor devices became available that the size of the equipment and the performance became acceptable.

In April 1997 the first Passive Millimeter Wave Imaging conference was held in Orlando as part of the SPIE Aerosense symposium. This was not the first time this topic had been covered at an SPIE conference but it was the first time that an entire conference had been dedicated to it. Although the location and name of the Symposium have changed over the years this conference has been run every year since 1997 with the addition of imaging millimeter wave radar in recent years. This was formally acknowledged in 2012 when the title was changed to "Passive and Active Millimeter Wave Imaging".

2017 marks the $20^{\text {th }}$ anniversary of the conference and this paper provides a historical review of some of the key contributions made during this period. Section 2 describes the first conference, section 3 the bibliometrics of the conference and sections 4, 5, 6 and 7 components, phenomenology and modeling, image enhancement and systems respectively before concluding in section 8 . For more details the reader is encouraged to consult the original manuscripts which can be found in the SPIE Digital Library ${ }^{i}$.

\section{FIRST CONFERENCE 1997}

This first conference ${ }^{1}$ was chaired by Roger Smith from Air Force Research Laboratories (USA) with co-chairs of David Gleed from the Defence Evaluation Research Agency (UK) and Scot Rogala from the Naval Air Warfare Centre (USA) and had 26 papers. The conference consisted of four sessions, Systems, Components, Modeling and Phenomenology and Resolution enhancement.

\subsection{Systems}

The conference began with a paper by Ewen on the Millimeter-wave Analysis of Passive Signatures (MAPS) which was a data collection exercise to support the development of smart tactical autonomous guidance systems. It collected data at

\footnotetext{
${ }^{\mathrm{i}}$ http://proceedings.spiedigitallibrary.org
}

*rappleby@innovasec.co.uk

Passive and Active Millimeter-Wave Imaging XX, edited by David A. Wikner, Duncan A. Robertson, Proc. of SPIE Vol. 10189, $1018902 \cdot$ (c) 2017 SPIE · CCC code: 0277-786X/17/\$18 · doi: 10.1117/12.2262476 
35, 60 and $90 \mathrm{GHz}$ from a mobile platform and images were shown of terrain and landmines under snow. The second paper presented by Yujiri described progress on the prototype passive millimeter wave camera operating at $89 \mathrm{GHz}$ with a field of view of $15 \times 10$ degrees, an 18 inch aperture and a frame rate of $17 \mathrm{~Hz}$. The unique thing about this camera was that it utilized a 26 × 40 focal plane of direct detection gallium arsenide Monolithic Microwave Integrated Circuits (MMICs). Imagery was presented from a prototype system having a single line of receivers ( $1 \mathrm{x} 40)$ which demonstrated an NEDT of $1.8 \mathrm{~K}$. The third paper by Gleed discussed surveillance, the merits of operating at different frequencies and presented state of the art imagery from a $94 \mathrm{GHz}$ system using 8 heterodyne receivers and a $1.2 \mathrm{~m}$ Cassegrain antenna. Two papers were presented on concealed weapon detection both using $94 \mathrm{GHz}$ heterodyne technology with Hugenin presenting their polarization twist reflector and also discussing a through-wall active system. Pergande presented a breadboard system with a $30 \mathrm{~cm}$ lens and some early imagery showing the detection of a gun through clothing. He also advocated combining this system with an infrared system. Finally Kreis described a millimeter and sub-millimeter wave sounder for space borne earth observation.

\subsection{Components}

Weinreb described MMICs using both gallium arsenide and indium phosphide substrates with gate lengths of 0.1 and $0.25 \mu \mathrm{m}$. The pros and cons of heterodyne or direct detection were discussed but the paper concluded that whatever architecture was chosen MMICs could be used to construct very compact and low cost passive receivers. Kuroda discussed the gallium arsenide $0.1 \mu \mathrm{m}$ HEMT MMICs that had been used in the focal plane array imager described in section 7.1.1. Harvey described an up-conversion method which modulated the signal from an array of passive millimeter wave receivers onto an optical fiber and carried this to a lens where the fiber geometry mimicked the geometry of the passive millimeter wave array with the light being imaged onto a video camera. Salmon reported on the absolute temperature stability of radiometers concluding that changes in the instrument temperature could produce significant changes in the system noise temperature and recommended improvements to components to overcome this, particularly the detector and amplifier. Rahman described work on uncooled micromachined microbolometers consisting of a thin niobium film on a silicon nitride membrane positioned at the base of a horn etched in silicon. An electrical NEP of $8.3 \times 10^{-11} \mathrm{~W} / \sqrt{ } \mathrm{Hz}$ was observed. Manasson reported work on a novel spinning grating antenna for a millimeter wave imaging system. The antenna operated at $94.3 \mathrm{GHz}$ and used a dielectric rod with a rotating metal grating to scan the beam. Denisov described the possibility of designing an array of receiving elements which used both Josephson junctions and cooled FET or HEMT amplifiers.

\subsection{Modeling and Phenomenology}

Falcone compared the theoretical and experimental estimates of surface microwave emissivity by taking data from the MAPS sensor at 35, 60 and $95 \mathrm{GHz}$ and comparing it to the EMITS computer model which is based on the Fresnel equations and reported excellent agreement. Blume described the IRMA model which has a passive millimeter wave channel as well as infrared, ladar and radar and comparisons were made between IRMA output and data from the Millitech sensor data collected from a landmine test area. Williams described MACET which along with IRMA had been used to model MAPS data. Albers discussed the relevance of coherent versus incoherent modeling of the radiance from a buried object and concluded that an incoherent model should be more accurate. Hauss described the Advanced Radiometric Millimeter-Wave scene Simulation (ARMSS) code which provided comprehensive end-to-end scene simulation based on "first-principles". ARMSS was developed to support the camera project described in section 7.1.1. ARMSS was validated against real data and also used to provide input to a flight simulator. Eden investigated the feasibility of autonomous guidance from passive millimeter wave data collected in a separate activity. Andreyev compared the brightness temperature of predicted and measured data at $35 \mathrm{GHz}$ for an airborne sensor. Visible imagery was also used to derive millimeter wave brightness temperatures which were compared to their real signatures.

\subsection{Resolution Enhancement and Super-resolution}

Lettington described a fast non-linear algorithm similar to that of Gerchberg and reported enhancing passive millimeter wave images beyond their diffraction limit. Pang reported an iterative Maximum Likelihood algorithm the main aim of which was to enhance imagery from the passive millimeter wave element of a multispectral seeker. Reeves reported on the difficulties of applying super-resolution and concluded that image processing alone offers little promise for significant super-resolution. Pirogov described different super-resolution algorithms including Regularization and the Method of Reduction describing the latter as a method of correcting the data by using a hypothetical model of the system to derive the point spread function. 


\subsection{Summary}

In this first conference it was clear that the field was growing and moving into a new phase as MMICs began to unlock the possibility of a focal plane array. There was no shortage of innovative systems designs which included systems folded using polarization, lenses and phased arrays with each having different strengths and weaknesses. The system parameters were traded off to try and deliver good spatial resolution and thermal sensitivity in real time at a cost that was affordable. The basic understanding of the phenomenology was there but initially the amount of experimental data was small making it difficult to quantify any models. It should also be remembered that at this time digital computing and signal processing were advancing rapidly and in some cases was still limiting performance.

\section{BIBLIOMETRICS}

In 20 years 466 papers have been submitted with 25 countries being represented. The number of papers produced by country is shown in Figure 1 and the institutions which have produced more than ten papers in this period are listed in Table 1.

35 and $94 \mathrm{GHz}$ were the main operating frequencies but more recently sub-millimeter wave systems have been reported.

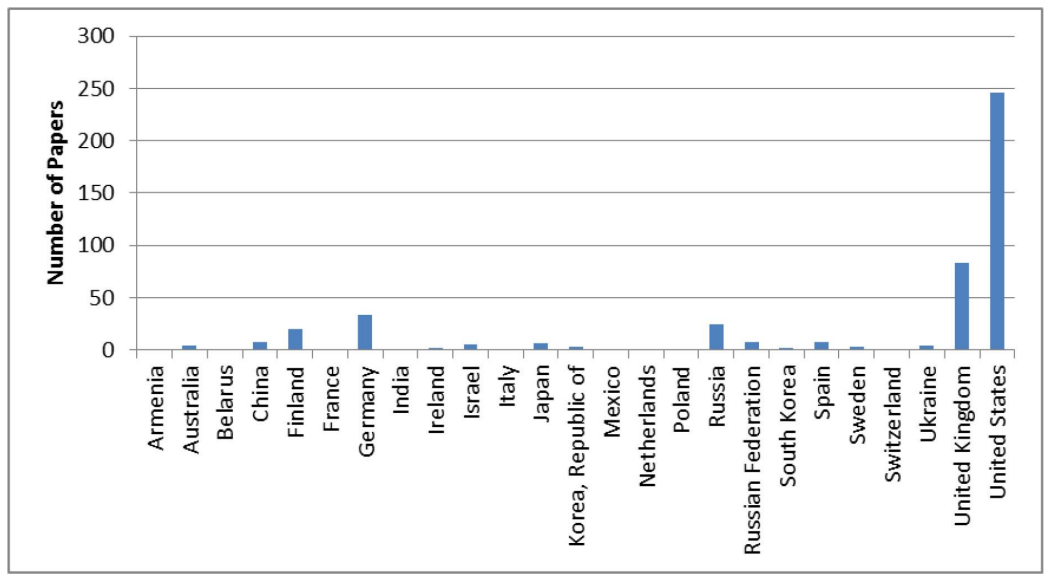

Figure 1 Papers by country

\begin{tabular}{|l|}
\hline Institutions \\
\hline Army Research Laboratory \\
\hline Moscow State University \\
\hline National Institute of Standards \\
\hline Pacific Northwest \\
\hline QinetiQ \\
\hline Trex Enterprises \\
\hline TRW \\
\hline University of Reading \\
\hline
\end{tabular}

Table 1 Institutions which produced more than 10 papers

It is difficult to identify trends from the keywords provided by authors as they are not harmonized - that is different authors have used the same keyword to mean something completely different. However it is possible to subjectively identify some trends. Certainly, there has been a steady move towards higher frequencies, this being driven by the technology available.

In 1997 there was an interest in stand-off systems which could operate from aircraft or other platforms and this continued as the performance in poor weather was exploited. There were also two papers addressing concealed weapon detection and there was a steady trickle of papers on this topic over the next few years which eventually became a major contribution to the conference. The work being conducted on imaging radars for concealed weapon detection was also reported at the conference some years later and added to the increasing number of papers on this topic.

The conference has had a steady stream of papers on image enhancement including image calibration and superresolution. In the case of super-resolution the aim was to improve the spatial resolution of the image to try and compensate for the relatively long wavelength when compared to the infrared part of the spectrum using algorithms.

The community has benefitted from an ever increasing understanding of the phenomenology arising from both experimental measurements and modeling activities with a great deal more being known about materials and the environment than was known in 1997.

\section{COMPONENTS}

In 1997 the first MMICs for radiometry were reported and indeed were being developed by TRW (Northrop Grumman) for their camera (see section 7.1.1). For the rest of the community who did not have access to these devices heterodyne 
detection using mixers with planar diodes was the technology of choice. MMIC technology along with its packaging using both gallium arsenide and indium phosphide matured so that it could be used in systems initially at $35 \mathrm{GHz}$ and then later at $94 \mathrm{GHz}$ (see section 7.1.2). For radiometry the next major breakthrough came when high sensitivity diodes were introduced in $2006^{2}$ as this reduced the amount of pre-detector gain from $\sim 60 \mathrm{~dB}$ to $\sim 30 \mathrm{~dB}$ which in turn meant a radiometer at $\mathrm{W}$-band could be made with just two chips. There is no doubt that as processes mature the frequencies at which MMICs can operate will increase and a prototype MMIC receiver at $670 \mathrm{GHz}$ was recently reported ${ }^{3}$. SiGe BiCMOS $94 \mathrm{GHz}$ radiometer chipsets were reported at W-band in $2013^{4}$ and whilst not having the same performance as GaAs or InP offer the advantage of low cost production. The arrival of the cooled micro-bolometer in 2007 provided a route to large focal plane arrays at high frequencies, albeit at the cost of cooling to cryogenic temperatures. Whilst it can only be a matter of time before MMICs catch up and achieve the same performance at room temperature as these cryogenic systems it will depend on the application as to which is most likely to succeed.

Much of the increase in the development of radar systems at millimeter and submillimeter wave frequencies has been enabled by the technological improvements in amplifier, multiplier and mixer devices. Notably, the improvement in GaAs Schottky barrier diode technology from the likes of VDI, JPL, Chalmers and RAL has yielded varactor diode multipliers with high efficiency and high output power over wide bandwidths at frequencies well into the submillimeter range. The ability to drive multipliers with higher power at higher frequencies is being enhanced through the improvements in power amplifier technology, particularly with GaN. Greater integration of radar components is also continuing, resulting in more compact arrays of transceivers. A prime example of this integration is in the Rohde \& Schwarz QPS system (see section 7.3.3) which has utilized SiGe MMICs for much of its RF circuitry.

Whilst large focal plane arrays of detectors are feasible with cooled radiometric detectors, for many passive and active systems detector numbers remain limited and some form of beam scanning is required. Mechanical beam steering is still prevalent in many systems (QinetiQ passive ${ }^{5}$ see section 7.1.2, Asqella ARGON passive ${ }^{6}$, University of St Andrews IRAD radar $^{7}$, etc.) but its obvious limitations with regard to frame rate and field of view are driving the development of non-mechanical beam steering methods. Notable approaches are the all-electronic reconfigurable reflectarray (Agilent/Smiths see section 7.3.2) suitable for lower frequencies, the electrically controlled MEMS reconfigurable reflectarrays fabricated on silicon wafers (Aalto/VTT) for use in the submillimeter range $^{8}$, and the millimeter / submillimeter optically controlled photo-injected Fresnel zone plate antenna, piFZPA ${ }^{9}$, (St Andrews). Currently, no prime solution exists and much future work in this area is anticipated.

An alternative scanning method for radar was described in our first conference in 1997 and consisted of a spinning grating antenna ${ }^{10}$. A drum with a metal grating is rotated next to a dielectric waveguide which causes the beam to scan in the plane of the dielectric waveguide. This type of antenna formed the basis of one of the radars which were evaluated for seeing in Brownout caused by helicopter rotor downwash.

\section{MODELING AND PHENOMENOLOGY}

In 1997 the understanding of the detail of target and background signatures was limited mainly due to a lack of good experimental data and ground truth. Initially modeling attempts were limited either to taking infrared or visible models and modifying them for the millimeter wave region or creating models from scratch (see section 2.3). In both cases the results were encouraging but it was clear further work needed to be done. There has now been a great deal of data collection including polarimetric data of scenes ${ }^{11-13}$, dust ${ }^{14}$, skin $^{15}$ plus many others and our understanding of materials in the millimeter and sub-millimeter wave region has greatly improved. Modeling has also improved considerably, benefitting from high power computers and digital cinematography giving rise to realistic models of objects, people and scenes. When this is combined with models that account for the refractive index of materials and their bidirectional reflectivity distribution function (BRDF) the modelled results can be very similar to the experimental results ${ }^{16}$.

\section{IMAGE ENHANCEMENT}

Over the last 20 years image enhancement has been mentioned $\sim 200$ times in the keywords of papers. This refers to either algorithms used to improve image quality and remove artefacts using interpolation and calibration techniques or super-resolution. Systems using large numbers of receivers require algorithms to replace dead elements and conduct calibration to ensure each element has the same response. Many systems utilize these methods however the details are often not presented in the public domain. 
Super-resolution is a technique that can in principle generate high spatial frequencies beyond the pass band in the restoration of a band limited image. An excellent review of these methods with some examples of what can be achieved was given by Lettington ${ }^{17}$ and the results for the Lorentzian method are shown in Figure 2.

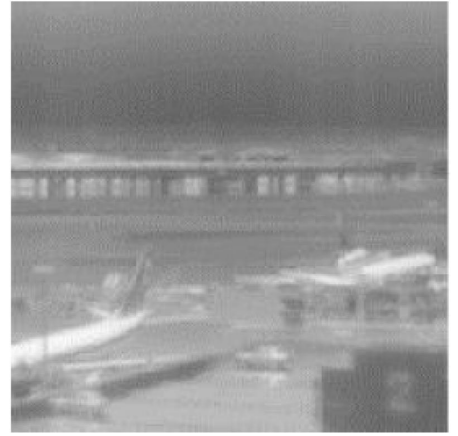

Figure 2 a Original $94 \mathrm{GHz}$ image of Heathrow

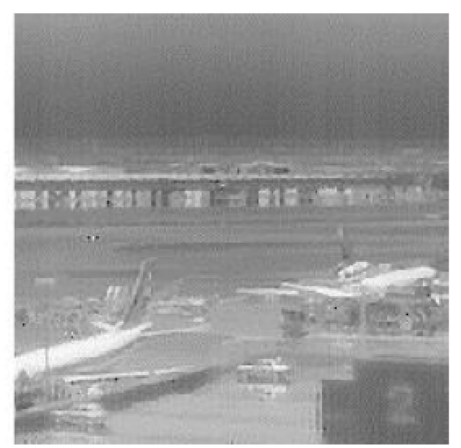

b. Image super-resolved using two-dimensional Lorentzian method.

The advantages are very limited when data from real systems is used and to the best of the authors' knowledge these techniques are not widely deployed. This was predicted by Reeves in $1997^{18}$ who after reviewing the limitations of super-resolution concluded that the commonly used prior information has some ability to accomplish super-resolution but this ability is fundamentally limited. On the other hand, prior information can be quite useful in extending the effective bandwidth in the measured spatial frequency region, due to the noise averaging effect that prior information has in the frequency domain. In his view image processing alone held little promise for significant super-resolution. He further concluded that optimizing system performance to increase the SNR at higher spatial frequencies could provide more benefit.

\section{SYSTEMS}

Early passive millimeter wave imaging systems utilized heterodyne receivers benefitting from the improved performance in planar diode technology. However this approach constrains the number of receivers you can accommodate as you have to provide a local oscillator signal to each mixer. The arrival of MMICs initially at 35 and then at $94 \mathrm{GHz}$ provided a means of using direct detection by amplifying at the RF frequency and then directly detecting the signal with a diode. This much simpler architecture allowed larger focal planes to be manufactured and a local oscillator was not required. A further advantage derived from the excellent thermal sensitivity of both heterodyne and direct detection receivers is that they can be used to image more than one point in the scene in a single frame. This has given rise to several elegant solutions, including rotating scanners, phased array beam formers and reflectarrays.

Millimeter wave imaging radar systems followed a similar progression but as they use a transmitter they benefit from much higher signal to noise ratio and so fewer receivers are needed to cover the field of view. Perhaps the most successful imaging radar in the last 20 years has been the holographic portal system operating at $26 \mathrm{GHz}$ based on linear arrays of rotating transceivers which has been commercialized and deployed in many airports ${ }^{19}$. A second system using a reflectarray operating at $24 \mathrm{GHz}$ has also achieved success ${ }^{20}$.

In recent years we have begun to explore the sub-millimeter wave region as MMICs have moved to even higher frequencies and mixers and multipliers have become available. These developments have made it possible to build radars at $670 \mathrm{GHz}$ for stand-off detection.

Cooled bolometers through their use of very simple receive elements have provided a route to large focal plane arrays with 100s of receivers, albeit at the expense of having to cool them to low temperatures. These systems currently hold the record for the imagery with the highest thermal sensitivity.

Some examples of these achievements taken from this conference series are given below.

\subsection{Direct Detection Focal Plane arrays}

\subsubsection{TRW Camera}

The primary mission of this 1990 s era camera was to demonstrate the utility of passive millimeter wave imaging to serve as an auxiliary sensor for pilots during landing and taxiing at airports in poor visibility. To achieve this aim the camera 
required a $17 \mathrm{~Hz}$ frame rate, an NEDT of $4 \mathrm{~K}$ and instantaneous field of view of $15 \mathrm{H} \mathrm{x} 10 \mathrm{~V}$ degrees. The camera ${ }^{21}$ shown in Figure 3 used $89 \mathrm{GHz}$ MMICs with a10 GHz bandwidth and used an 18 inch Rexolite ${ }^{\mathrm{TM}}$ lens and a dithering mirror inclined at 45 degrees to sample the imagery at the Nyquist limit. The focal plane consists of 1040 receiver channels with each consisting of a linearly tapered slot antenna and 4 GaAs MMIC amplifier chips using $0.1 \mu \mathrm{m}$ pseudomorphic HEMT technology. The receivers themselves achieved a thermal resolution of $0.9 \mathrm{~K}$ which gives an indication of the losses associated with the optical path. Calibration of the focal plane was achieved with a millimeter wave source integrated within the optical sub-system. The system was flight tested in several types of landing and surveillance modes and remains an important benchmark in the development of FPA-based millimeter wave imaging systems.

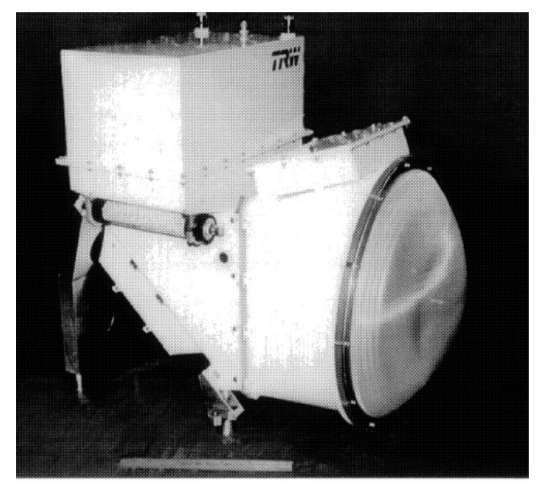

a. Imager

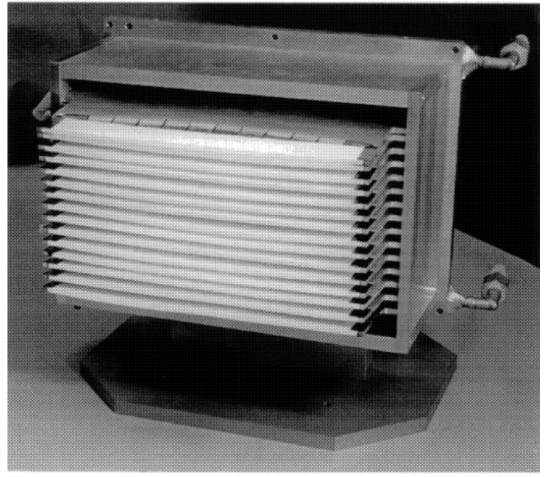

b. Focal Plane of 13 cards each with 40 detectors

Figure 3 TRW Focal Plane array camera

\subsubsection{DERA (QinetiQ) Imagers}

The first of a series of imagers using a linear array of direct detection receivers and an optical system folded using polarization and producing real time real time imagery was initially reported in 2002 . It operated at $35 \mathrm{GHz}$ and the optical design $^{22}$ is shown in Figure 4.

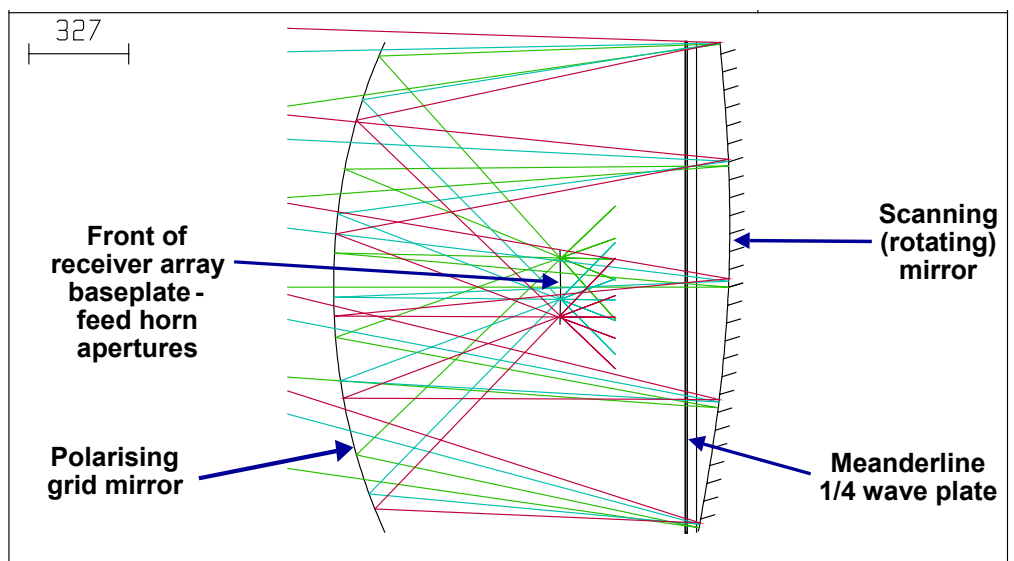

a Folded optical design

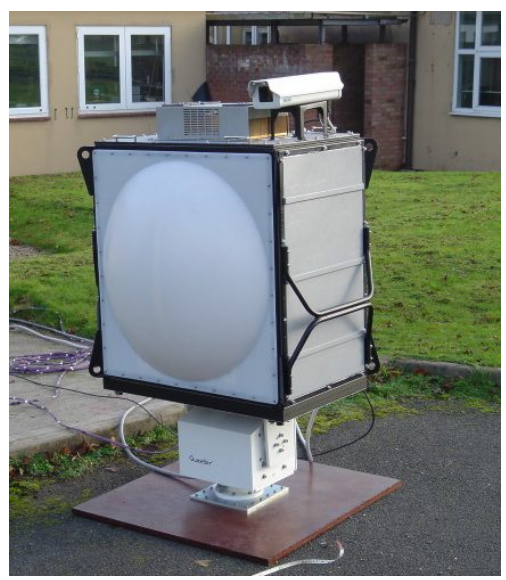

b. $94 \mathrm{GHz}$ security scanner

Figure 4 Imagers using polarization to fold the optical design

It consists of a curved aspheric polarizing grid as the focusing element, a linear array of receivers plus feed horns, a meanderline quarter-wave plate to rotate the plane of polarization and a rotating tilted mirror with an aspheric concave profile as a scanner. Millimeter wave radiation from the target (coming from the left-hand-side) enters through the polarizing grid and is polarized by it. The radiation then passes through the meanderline, which converts it into circular polarization. On reflection from the rotating tilted scanner, which produces the conical scan, the circular polarization changes handedness before passing back through the meanderline, where it is converted back to linear polarization but 
orthogonal to the incoming polarization due to the handedness change at the scanner. When this radiation reaches the polarizing grid, it is now reflected and focused down onto the receiver array.

Several variants of this design were produced for concealed weapon detection ${ }^{23}$ as shown in Figure $4 \mathrm{~b}$, seeing in degraded visual environments ${ }^{24}$ and scanning trucks for illegal immigrants ${ }^{25}$.

\subsubsection{Millitech/Millivision scanned system}

The initial optical design of the Millitech camera ${ }^{26}$ also used polarization folding and is shown in Figure 5. The polarization components are the twist reflector and transreflector. In this imager a lens and a linear array of double sideband mixers were used. There were several variants developed from the 1997 design shown below over the years for concealed weapon detection and remote sensing ${ }^{27}$.

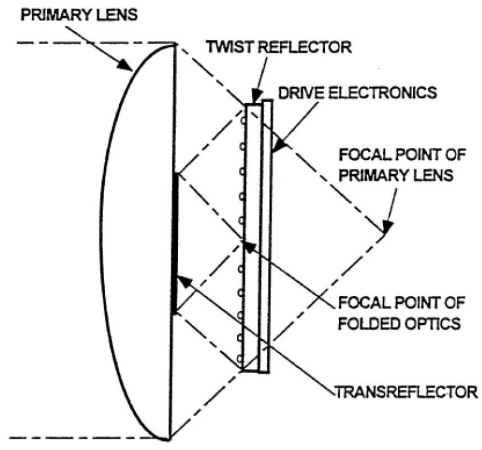

a. Coaxial folded optics

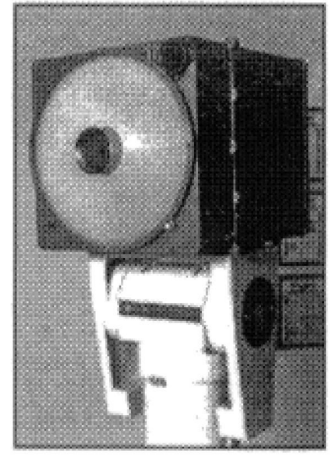

b. Prototype video surveillance camera

Figure 5 Millitech camera

\subsubsection{Trex Imager}

The novel concept of the TREX imager $^{28}$ reported in 1997 used a video camera to form the final image as shown in Figure 6. This pupil-plane antenna imager used 36-inch long vertical slotted waveguides to frequency scan in the vertical direction and aperture synthesis in the horizontal direction. This was achieved by injecting the amplified down-converted signals into a multi-channel Bragg cell which when illuminated with a laser and imaged with a lens produced an image through optical beam forming. A major advantage of this concept was that it used optics to directly form the image which is a replica of the incident millimeter wave signal.

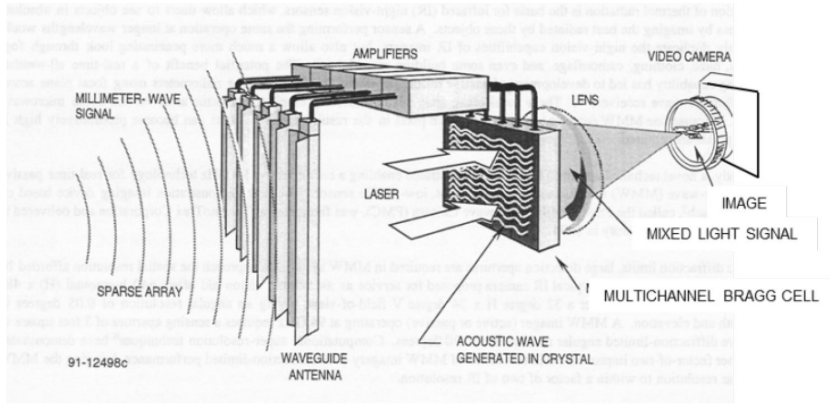

a. Imaging Concept

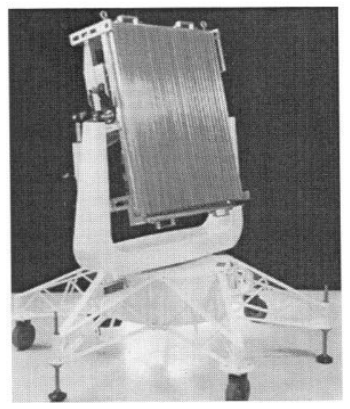

b. $94 \mathrm{GHz}$ Imager

Figure 6 TREX Imager $94 \mathrm{GHz}$

A later version of the system maintained the pupil plane approach but used a new antenna and processor design and greatly improved performance ${ }^{29}$. The 32 -element waveguide array was replaced with a 2-foot square planar dielectric antenna. The same beam steering concept was used whereby vertical field of regard (FOR) was achieved by frequency scanning and horizontal FOR by phase processing. The processor for this system was built using numerous Rotman lenses that sorted out the signals which were eventually detected with Sb-heterostructure diodes. The system produced 
passive $\mathrm{mmW}$ images over a $30 \times 24$ degree FOR with 0.35 -degree angular resolution and a $2 \mathrm{~K}$ temperature resolution at a $30-\mathrm{Hz}$ frame rate. The system also went on to undergo helicopter flight trials.

\subsubsection{Phase Sensitive Innovations}

The imaging concept proposed by Phase Sensitive Innovations in 2008 was to upconvert the received MMW signals to the optical sidebands of a telecommunications laser carrier right in the aperture plane directly behind the antenna ${ }^{30}$ as shown in Figure 7 and then to use optics to do the beam forming and detection. This concept has several advantages one of which is that optical fibers can be used to transport the signal and so the processor can be some distance from the array. The barrier to success was initially developing efficient lithium niobate electro-optical modulators to convert millimeter wavelength radiation to optical wavelengths. Another significant hurdle involved devising a method of phase balancing the optical fiber channels. Work was originally started at $35 \mathrm{GHz}$ for proof of concept and was later demonstrated at $77 \mathrm{GHz}$. A 220-channel, $77 \mathrm{GHz}$ system was developed with a 20 x 20 degree field of regard, angular resolution of 0.4 degrees, $25 \mathrm{~Hz}$ frame rate and NETD of $2 \mathrm{~K}$. The system was flight tested and used to measure the attenuation of desert dust. They are working towards a $95 \mathrm{GHz}$ system to decrease the size of the system and innovative liquid crystal polymer ${ }^{31}$ are being used in the modulator .

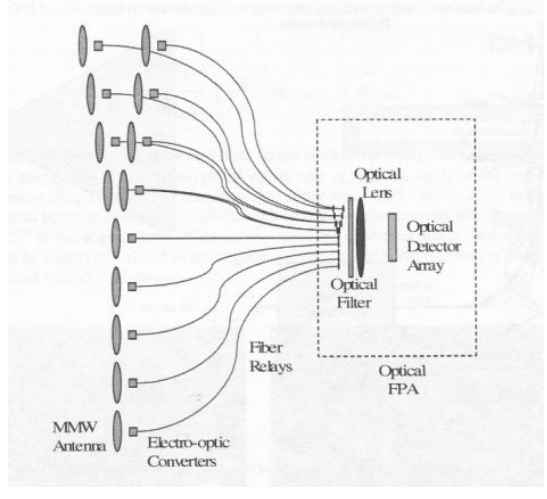

a. Upconversion Imaging Concept

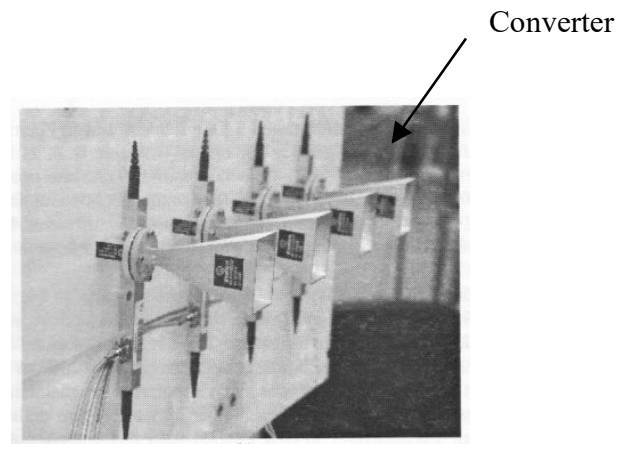

b. $\quad 35 \mathrm{GHz}$ Prototype testing

Figure 7 Phase Sensitive Innovations upconversion concept

\subsection{Cooled Systems}

By 1997 bolometers had already made an impact on thermal imaging at infrared wavelengths and were also being developed for radio astronomy. In our first conference in 1997 a paper was presented on an uncooled bolometer for passive millimeter wave imaging. However, operating at room temperature produced too much background noise and despite the opportunity for large focal planes the required thermal sensitivity could not be achieved and this looked very much like a dead end. However the use of cooled microbolometers utilizing superconductors and low temperatures achievable with closed cycle helium cryostats provided the necessary route to the required thermal sensitivity.

\subsubsection{NIST and VTT}

This type of system was first reported at this conference in $2007^{32}$ although at that time the detector technology shown had already been previously reported. Those devices were capable of a Noise Equivalent Temperature Difference (NETD) of $125 \mathrm{mK}$ over a pre-detection bandwidth from $0.2-1 \mathrm{THz}$ using a post-detection integration time of $30 \mathrm{~ms}$. Further improvements in devices were aimed at reducing this number to a few tens of $\mathrm{mK}$. Such an exquisite sensitivity was considered necessary in order to achieve the stringent requirements for low false positive alarm rate combined with high probability of detection needed for concealed weapon detection. This technological approach allowed for excellent per frame system NETD (objective $0.5 \mathrm{~K}$ or below at $30 \mathrm{~Hz}$ frame rate), and was also amenable to multispectral (color) imagery that enhances the discrimination of innocuous objects against real threats. This imaging system utilized superconducting ultrawide band antenna-coupled microbolometers, coupled to innovative room temperature read-out electronics, and operated within a cryogen-free pulse tube refrigerator. Initially 8 elements were proposed with plans to increase this to 128 elements and adopt a conical scan philosophy. 
The larger linear arrays gave rise to a series of imagers including the IMSK imager shown in Figure 8 below with some imagery. This technology is currently being used commercially by Asqella ${ }^{\text {ii }}$ in their ARGON imager.
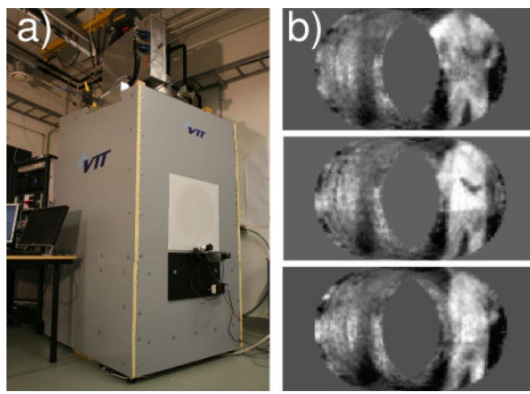

(a) IMSK imager at VTT and (b) its imagery. (c) is from a single channel imager using the same detector technology

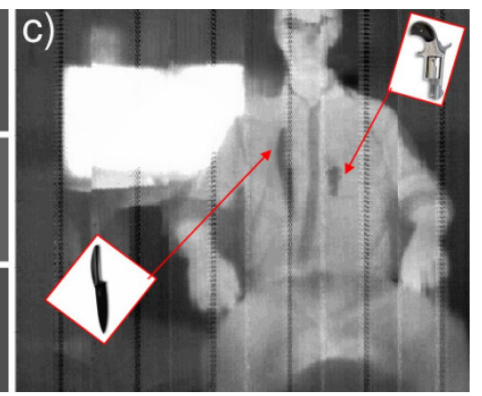

(d) Superconducting microbolometer

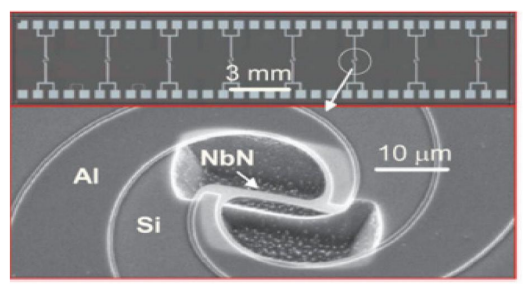

Figure 8 Cooled microbolometers

\subsubsection{Jena}

The IPHT SAFE-VISITOR ${ }^{33}$ is a $350 \mathrm{GHz}$ passive imaging system demonstrator shown in Figure 9 is based on deepcryogenic spider web bolometers operated at around $0.3 \mathrm{~K}$ and was reported in 2010 . Due to the very low operating temperature it is photon-noise limited and can outperform any other existing passive system with respect to radiometric resolution. The unit uses a cryogen free pulse tube ${ }^{4} \mathrm{He} /{ }^{3} \mathrm{He}$ evaporative refrigerator. The 20-channel bolometer FPA images the subjects through a Cassegrain telescope with a scanning secondary mirror. The FoV of the system is circular with $\sim 1 \mathrm{~m}$ diameter at $8.5 \mathrm{~m}$ focus distance. The system has a spatial resolution of $\sim 2 \mathrm{~cm}$, and a NETD of $0.4 \mathrm{~K}$ at 10 fps.

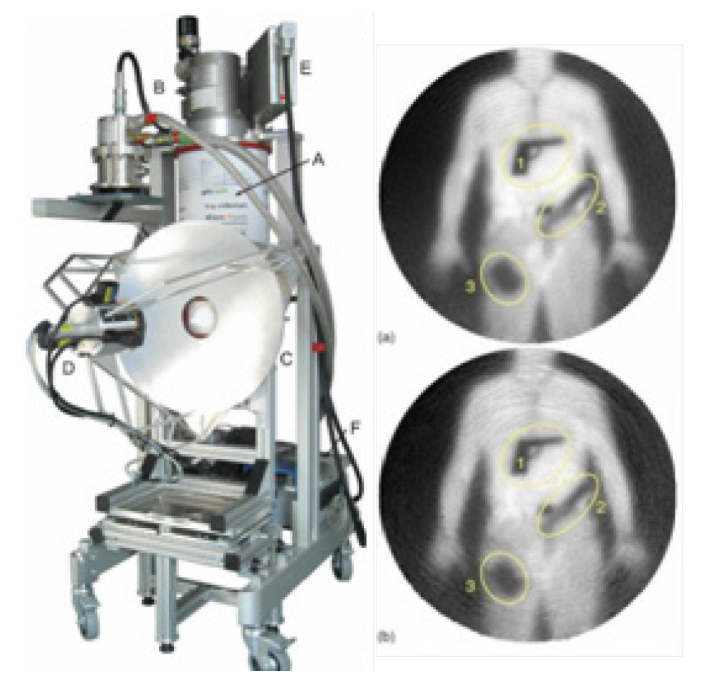

Figure 9 (Left) The SAFE-VISITOR system (compressor \& vacuum pumps not shown). (Right) Image of person with concealed items at $1 \mathrm{~Hz}(\mathrm{a})$ and $10 \mathrm{~Hz}(\mathrm{~b})$ frame rate.

\subsection{Radar Imaging}

Whilst the conference initially focused on passive millimeter wave imaging it has in fact included radar activities since $1999^{34}$. The first radar work was on a dual-mode 'radarometer' developed by Millitech ${ }^{\text {iii }}$ for AFRL. AFRL also sponsored work at that time on combining passive millimeter wave imaging with LIDAR imaging, in conjunction with the University of Missouri. The number of radar papers in the conference started to grow from about 2005 onwards and

\footnotetext{
ii https://asqella.com/

iii http://www.millitech.com/
} 
the conference title added the term 'active' in 2012 to reflect this trend. Most of the radar papers have been concerned with concealed object detection or security screening but have also included material inspection, volcano imaging and helicopter landing in brownout conditions. In the last decade there has been a distinct move to research higher frequencies for radar systems, typically 220,340 and $670 \mathrm{GHz}$, which has been driven by a demand for high resolution and enabled by improved multiplier, mixer and amplifier device technology.

\subsubsection{Pacific Northwest National Laboratories}

The origins of what we now know as the L3 Provision ${ }^{\text {iv }}$ system shown in Figure 10 were first presented to the conference in $2000^{19}$. Operating in the $26-30 \mathrm{GHz}$ region the concept consists of vertical, high resolution, millimeter wave array of transceivers which is scanned in a cylindrical manner about the person under surveillance. Using a computer the image reconstruction is performed using synthetic aperture holographic methods and yields very high resolution imagery. Considerable effort has gone into improving the image quality and adding privacy software to this imager, much of it reported at this conference.

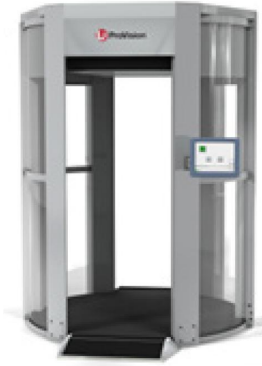

a. L3 Pro Vision

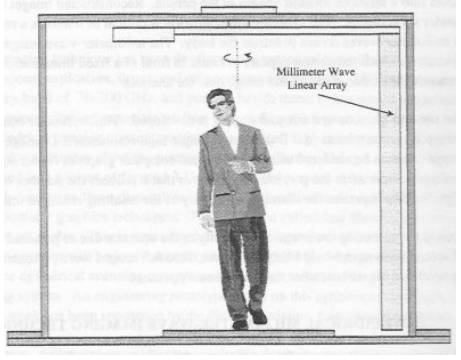

b. Initial concept

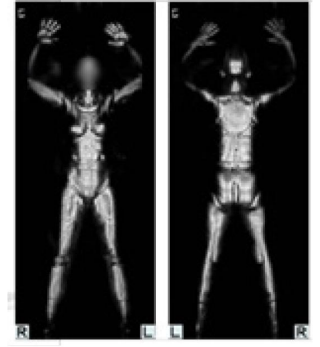

c. Imagery

Figure 10 L-3 Active millimeter wave portal.

\subsubsection{Agilent Imager / Smiths EQO}

This system which was acquired by Smiths and became known as EQO is shown in Figure 11. In this approach, developed by Agilent Technologies ${ }^{20}$ in 2006, beam scanning and image formation was carried out by reflecting a 24 $\mathrm{GHz} \mathrm{CW}$ transceiver off a digitally programmable reflectarray antenna which repeatedly focuses the beam to a series of points in a $3 \mathrm{D}$ volume at a rate of $10^{6}$ pixels/sec with no moving parts. Each "voxel" in the search volume corresponds to a specific phase pattern which is digitally programmed on to the reflectarray at very high speed. Thanks to the electronic scanning, only a few microwave components are required, and the digital beam control makes use of low cost microelectronics.

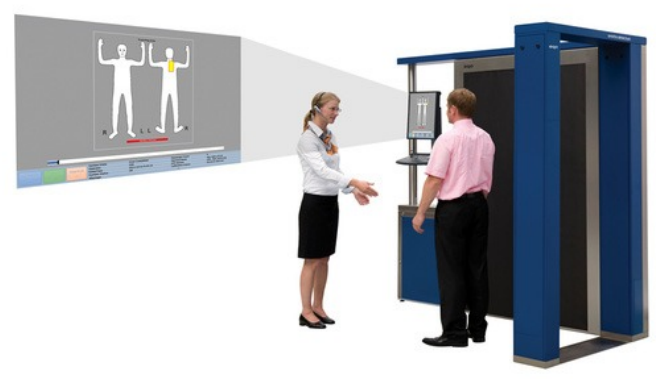

a. Smiths EQO ${ }^{\mathrm{v}}$

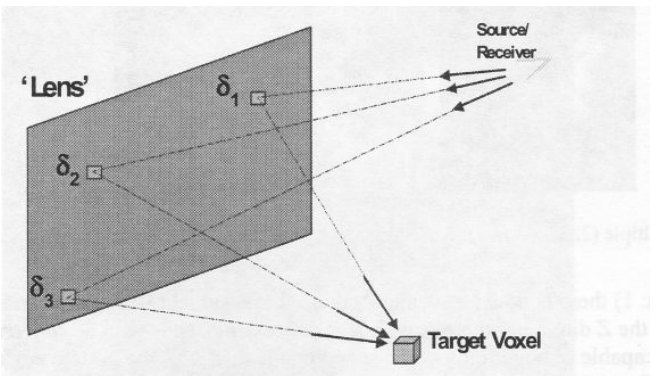

b. Imaging Concept

\subsubsection{Rohde and Schwarz}

Figure 11 Smiths Eqo and Imaging concept

The QPS system ${ }^{\mathrm{vi}}$ which is now being installed in significant numbers in airports was presented at the conference in $2013^{35}$. Building on advances in MMICs for the car collision avoidance radar market $(70-80 \mathrm{GHz})$ a portal which was a

\footnotetext{
${ }^{\text {iv }}$ http://www.sds.1-3com.com/advancedimaging/provision-2.htm

${ }^{v}$ https://www.smithsdetection.com/index.php?option=com k2\&view=item\&id=80:eqo\&Itemid=1420\#.WN5hFMm1vcs
} 
fully electronic solution delivering real-time imaging was reported. Furthermore, the continuously increasing capabilities of digital signal processing units allow for the utilization of digital-beamforming techniques for image reconstruction, thus offering new opportunities for imaging systems to use sophisticated operational modes. Based on these modern technologies, an advanced realization addressing personnel screening in E-band with planar multistatic sparse array design was reported.

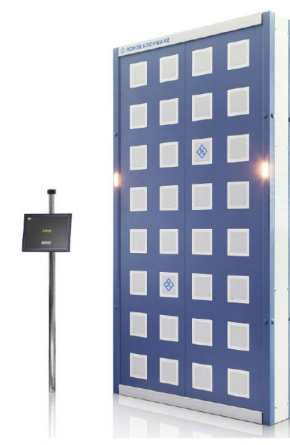

a. Operators console and flat panel phased array

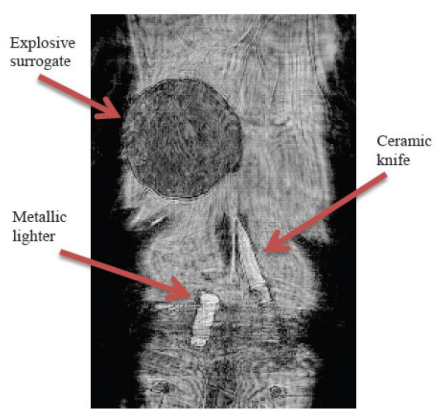

b. Image of a person concealing various objects.

Figure 12 QPS system

\subsubsection{Jet Propulsion Labs}

The Jet Propulsion Laboratory in 2011 reported on the impact of polarization diversity on their $670 \mathrm{GHz}$ 3D radar imager $^{36}$ which is perhaps the best known sub-millimeter wave imaging radar. The radar uses a heterodyne, double down-conversion FMCW architecture with $\sim 30 \mathrm{GHz}$ bandwidth chirps and software nonlinearity compensation to achieve $\sim 1 \mathrm{~cm}$ range resolution. The system has been designed to record very high spatial resolution images $(\sim 1 \mathrm{x} 1 \mathrm{x} 1$ $\mathrm{cm}^{3}$ voxels) at modest stand-off ranges (4 to $25 \mathrm{~m}$ ) and has successfully imaged weapons hidden under clothing.

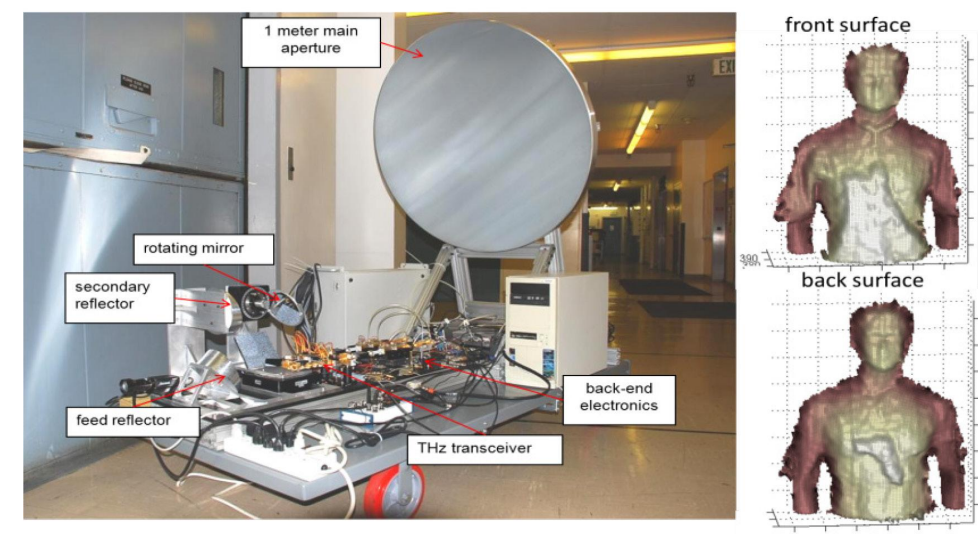

Figure 13 Jet Propulsion Laboratory $670 \mathrm{GHz} 3 \mathrm{D}$ radar imager with $\sim 1 \mathrm{x} 1 \mathrm{x} 1 \mathrm{~cm} 3$ resolution at $4 \mathrm{~m}$ range (right).

\section{CONCLUSIONS}

During the past 20 years we have seen passive imaging systems mature and be deployed for security, and loss prevention. Some of this success has been made possible by the development of low noise wide bandwidth high gain MMIC chips and innovations at the system level which have included mechanical scanning, polarization folding to reduce size, aperture synthesis and optical upconversion. More recently cooled microbolometers have provided a route to large focal planes with very high sensitivity. In line with system development we have also seen an improved understanding of the phenomenology and our ability to conduct system modelling and simulation.

\footnotetext{
${ }^{v i}$ https://cdn.rohde-schwarz.com/pws/dl_downloads/dl_common_library/dl_brochures_and_datasheets/pdf_1/QPS100200 bro en 3606-7160-12 v0200.pdf
} 
Imaging radar systems have also benefited from the developments in MMICs and have achieved even greater success and can be found today operating in many commercial airports. This success has also benefited from systems innovation particularly in phased array and reflectarray technology. More recently sub-millimeter wave radars enabled by developments in high frequency multipliers, power amplifiers and mixers are offering stand-off ranges of 10s of meters and also the possibility of high performance walk through systems.

Looking forward to the next 20 years is difficult but several observations can be made:

- Multimodal systems will be developed to give increased performance i.e. passive and active, infrared and millimeter wave

- There will continue to be greater integration between different elements of the systems

- This will include, RF and digital technology and their packaging and different sensing modalities

- There will be a steady increase in performance in passive systems as large focal planes start to become available

- Radars operating in the sub-millimeter wave region will provided stand-off security and or high fidelity portal type screening

- Whilst large 2D focal plane arrays of cooled detectors are emerging for passive imagers, the requirement for beam scanning will remain for uncooled radiometric detectors and radars. An increase in development activity of non-mechanical beam steering is anticipated.

The conference has provided an excellent forum for workers in the field to share their results and report progress across a wide range of projects and we can look back and consider this as a job well done! Looking forward there is still much to be done as we strive to meet ever more demanding requirements and the conference will continue to provide a forum for reporting this work.

\section{REFERENCES}

[1] Proc. SPIE. 3064, (1997).

[2] H. P. Moyer, R. L. Bowen, J. N. Schulman et al., "Sb-heterostructure diode detector W-band NEP and NEDT optimization," Proc. SPIE. 6211, 62110J-62110J-7 (2006).

[3] E. N. Grossman, K. Leong, X. B. Mei et al., "Passive $670 \mathrm{GHz}$ imaging with uncooled low-noise HEMT amplifiers coupled to zero-bias diodes," Proc. SPIE. 9078, 907809-907809-9 (2014).

[4] V. Jain, and F. Behbahani, "A SiGe BiCMOS W-band passive imaging receiver using lossless flicker-noise cancellation," Proc. SPIE. 8715, 871504-871504-5 (2013).

[5] R. N. Anderton, C. D. Cameron, J. G. Burnett et al., "Improvements to the design process for a real-time passive millimeter-wave imager to be used for base security and helicopter navigation in degraded visual environments," Proc SPIE. 9078, 907806-907806-17 (2014).

[6] S. Enestam, P. Kajatkari, O. Kivimäki et al., "Phenomenology of passive multi-band submillimeter-wave imagery," Proc SPIE. 9830, 98300B-98300B-8 (2016).

[7] D. A. Robertson, P. N. Marsh, D. R. Bolton et al., "340-GHz 3D radar imaging test bed with 10-Hz frame rate," Proc SPIE. 8362, 836206-836206-11 (2012).

[8] A. Tamminen, T. F. Gallacher, J. Ala-Laurinaho et al., "Developments of a reflectarray and its element characterization at millimeter wavelengths," Proc SPIE. 9078, 907807-907807-11 (2014).

[9] D. A. Robertson, T. F. Gallacher, R. Søndenå et al., "Millimeter wave imaging at up to 40 frames per second using an optoelectronic photo-injected Fresnel zone plate lens antenna," Proc SPIE. 9830, 983004-983004-10 (2016).

[10] V. A. Manasson, R. M. Mino, and L. S. Sadovnik, "Spinning grating antenna for MMW imaging," Proc. SPIE. 3064, 134-143 (1997).

[11] A. S. Hedden, D. A. Wikner, and R. W. Bradley, "Summary and analysis of $216 \mathrm{GHz}$ polarimetric measurements of in-situ rain," Proc. SPIE. 9462, 94620G-94620G-12 (2015).

[12] D. A. Wikner, "Polarimetric radiometry of natural scenes," Proc. SPIE. 4719, 391-396 (2002).

[13] D. A. Wikner, and G. Samples, "Polarimetric passive millimeter-wave sensing," Proc. SPIE. 4373, 86-93 (2001).

[14] D. A. Wikner, "Passive millimeter-wave imagery of helicopter obstacles in a sand environment," Proc. SPIE. 6211, 621103-621103-8 (2006). 
[15] R. A. Chamberlin, N. Mujica-Schwahn, and E. N. Grossman, "650 GHz bistatic scattering measurements on human skin." 9078, 90780B-907814 (2014).

[16] R. Appleby, H. Petersson, and S. Ferguson, "Concealed object stand-off real-time imaging for security: CONSORTIS," Proc SPIE. 9462, 946204-946204-11 (2015).

[17] A. H. Lettington, and N. E. Alexander, "Constrained image restoration applied to passive millimeter-wave images," Proc. SPIE. 5573, 334-343 (2004).

[18] S. J. Reeves, "Analysis of the difficulties and possibilities for superresolution," Proc. SPIE. 3064, 239-248 (1997).

[19] D. M. Sheen, D. L. McMakin, and T. E. Hall, "Combined illumination cylindrical millimeter-wave imaging technique for concealed weapon detection," Proc. SPIE. 4032, 52-60 (2000).

[20] P. Corredoura, Z. Baharav, B. Taber et al., "Millimeter-wave imaging system for personnel screening: scanning $10^{\wedge} 7$ points a second and using no moving parts," Proc. SPIE. 6211, 62110B-62110B-8 (2006).

[21] L. Yujiri, H. H. Agravante, M. Biedenbender et al., "Passive millimeter-wave camera," Proc. SPIE. 3064, 15-22 (1997).

[22] R. N. Anderton, R. Appleby, P. R. Coward et al., "Security scanning at 35 GHz," Proc. SPIE. 4373, 16-23 (2001).

[23] R. N. Anderton, R. Appleby, J. E. Beale et al., "Security scanning at 94GHz," Proc. SPIE. 6211, 62110C62110C-7 (2006).

[24] R. Appleby, P. Coward, and J. N. Sanders-Reed, "Evaluation of a passive millimeter-wave (PMMW) imager for wire detection in degraded visual conditions," Proc. SPIE. 7309, 73090A-73090A-8 (2009).

[25] R. Appleby, "The history of passive millimetre-wave imaging at QinetiQ," Proc. SPIE. 7117, 711702-711702-7 (2008).

[26] G. R. Huguenin, "Millimeter-wave video rate imagers," Proc. SPIE. 3064, $34-45$ (1997).

[27] T. D. Williams, "Environmental control for improved passive millimeter wave concealed object detection," Proc. SPIE. 8715, 87150D-87150D-7 (2013).

[28] R. Olsen, J. A. Lovberg, R.-C. Chou et al., "Passive millimeter-wave imaging using a sparse phased-array antenna," Proc. SPIE. 3064, 63-70 (1997).

[29] S. E. Clark, J. A. Lovberg, C. A. Martin et al., "Passive millimeter-wave imaging for airborne and security applications," Proc. SPIE. 5077, 16-21 (2003).

[30] J. P. Samluk, C. A. Schuetz, J. E. L. Stein et al., "Far field millimeter-wave imaging via optical upconversion." 6948, 694804-694804-10 (2008).

[31] R. D. Martin, S. Shi, Y. Zhang et al., "Video rate passive millimeter-wave imager utilizing optical upconversion with improved size, weight, and power," Proc. SPIE. 9462, 946209-946209-8 (2015).

[32] A. Luukanen, L. Grönberg, P. Helistö et al., "Passive Euro-American terahertz camera (PEAT-CAM): passive indoor THz imaging at video rates for security applications," Proc. SPIE. 6548, 654808-654808-7 (2007).

[33] E. Heinz, D. Born, G. Zieger et al., "Progress report on Safe VISITOR: approaching a practical instrument for terahertz security screening," Proc. SPIE. 7670, 767005-767005-8 (2010).

[34] D. G. Huddleston, J. Savage, B. M. Sundstrom et al., "Radarometer sensor: simultaneous active and passive imaging using a common antenna." 3703, 33-44 (1999).

[35] S. S. Ahmed, "Personnel screening with advanced multistatic imaging technology," Proc. SPIE. 8715, 87150B87150B-6 (2013).

[36] K. B. Cooper, R. J. Dengler, and N. Llombart, "Impact of frequency and polarization diversity on a terahertz radar's imaging performance," Proc. SPIE. 8022, 80220D-80220D-8 (2011). 